IJMMS 32:10 (2002) 615-625

PII. S0161171202112294

http://ijmms.hindawi.com

(c) Hindawi Publishing Corp.

\title{
INFINITE TWO-GENERATOR GROUPS OF CLASS TWO AND THEIR NON-ABELIAN TENSOR SQUARES
}

\author{
NOR HANIZA SARMIN
}

Received 24 December 2001

We classify all infinite 2-generator groups of nilpotency class two and determine their non-abelian tensor squares.

2000 Mathematics Subject Classification: 20F05, 20J99, 20 F99.

1. Introduction. For a group $G$, the non-abelian tensor square $G \otimes G$ of a group $G$ is generated by the symbols $g \otimes h, g, h \in G$, subject to the relations

$$
g g^{\prime} \otimes h=\left({ }^{g} g^{\prime} \otimes{ }^{g} h\right)(g \otimes h), \quad g \otimes h h^{\prime}=(g \otimes h)\left({ }^{h} g \otimes h h^{\prime}\right),
$$

for all $g, g^{\prime}, h, h^{\prime} \in G$, where ${ }^{g} g^{\prime}=g^{\prime} g^{\prime} g^{-1}$. The non-abelian tensor square is a special case of the non-abelian tensor product which has its origins in homotopy theory and was introduced by Brown and Loday in $[4,5]$.

In [3], Brown et al. started the investigation of non-abelian tensor squares as group theoretical objects. One of their main goals is the explicit computation of non-abelian tensor squares. The topic of this paper is the classification of infinite 2-generator groups of nilpotency class two and the determination of their non-abelian tensor squares. In [1, 7], this was done for 2-generator $p$-groups of class two, for $p$ an odd prime or $p=2$, respectively. Thus this paper completes the classification of 2 generator groups of class two and determination of their non-abelian tensor squares. For an overview of non-abelian tensor squares which have been determined, we refer to [6] and also to [2], where infinite metacyclic groups were classified and their non-abelian tensor squares determined.

2. The classification. In this section, we classify the infinite 2-generator groups of nilpotency class two up to isomorphism. As a preliminary step, we classify the above groups which are split extensions of a $p$-group by an infinite cyclic group.

Proposition 2.1. Let $G=\langle a, b\rangle$ be a 2-generator group of nilpotency class less than or equal to 2 of the form $G=P \rtimes\langle b\rangle$, where $\langle b\rangle$ is an infinite cyclic group, and $P=\langle[a, b]\rangle\langle a\rangle$ is a p-group. Then $G$ is isomorphic to exactly one group of the following types:

$$
G \cong(\langle a\rangle \times\langle c\rangle) \rtimes\langle b\rangle,
$$

where $[a, b]=c,[a, c]=[b, c]=1,|a|=p^{\alpha},|c|=p^{\gamma}, \alpha \geq \gamma \geq 1$;

$$
G \cong\langle a\rangle \rtimes\langle b\rangle,
$$


where $[a, b]=a^{p^{\alpha-\gamma}},|a|=p^{\alpha}, \alpha \geq 2 \gamma \geq 2$;

$$
G \cong(\langle a\rangle \times\langle c\rangle) \rtimes\langle b\rangle,
$$

where $[a, b]=a^{p^{\alpha-\gamma}} c,[c, b]=a^{-p^{2(\alpha-\gamma)}} c^{-p^{\alpha-\gamma}},|a|=p^{\alpha},|c|=p^{\sigma}, \gamma>\sigma \geq 1, \alpha+\sigma \geq$ $2 \gamma$;

$$
G \cong\langle a\rangle \times\langle b\rangle,
$$

where $[a, b]=1,|a|=p^{\alpha}$.

The groups in the above list have nilpotency class two precisely for (2.1), (2.2), and (2.3), and are abelian for (2.4).

Proof. Let $G$ be a group as in the hypothesis. Since $G$ is nilpotent of class less than or equal to $2, P$ is abelian. Suppose first $[a, b]=1$. Obviously, $G$ is of type (2.4). Now, let $[a, b] \neq 1$. First, let $\langle[a, b]\rangle \cap\langle a\rangle=\langle 1\rangle$. Set $[a, b]=c$. This implies that $P=\langle c\rangle \times\langle a\rangle$. Thus $G$ is the group of type (2.1). Next, let $\langle[a, b]\rangle \cap\langle a\rangle \neq\langle 1\rangle$. If $\langle[a, b]\rangle \subseteq$ $\langle a\rangle$, then evidently, $G$ is the group of type (2.2). Finally, suppose that $\langle[a, b]\rangle \cap\langle a\rangle$ is a proper subgroup of $\langle[a, b]\rangle$. We have $1=[a, b]^{p^{\gamma}}=\left[a, b^{p^{\gamma}}\right]$. This implies that $b^{p^{\gamma}} \in Z(G)$, thus $\left\langle b^{p^{\gamma}}\right\rangle \triangleleft G$. Therefore, $G /\left\langle b^{p^{\gamma}}\right\rangle$ is isomorphic to $H=(\langle u\rangle \times\langle v\rangle) \rtimes\langle w\rangle$ where $[v, w]=v^{p^{\alpha-\gamma}} u,[u, w]=v^{-p^{2(\alpha-\gamma)}} \cdot u^{-p^{\alpha-\gamma}},|v|=p^{\alpha},|w|=p^{\beta},|[v, w]|=p^{\gamma}$, $\alpha, \beta, \gamma, \sigma \in \mathbb{N}, \gamma>\sigma \geq 1, \alpha+\sigma \geq 2 \gamma, \beta \geq \gamma$, as follows from [1, Theorem 2.4] for $p$ odd and [7, Theorem 2.5] for $p=2$, respectively.

It is easy to show that the nilpotency class of each group of type (2.1), (2.2), and (2.3) is two and they are all pairwise nonisomorphic.

Now, we are ready to state and prove the main theorem of this section. Note that $F_{2} / \gamma_{3}\left(F_{2}\right)$ is the free group of rank 2 and class 2 , and is denoted by $\mathcal{H}$, known as the Heisenberg group.

THEOREM 2.2. Let $G$ be an infinite non-abelian 2-generator group of nilpotency class two. Then $G$ is isomorphic to exactly one group of the following types:

$$
G \cong(\langle a\rangle \times\langle c\rangle) \rtimes\langle b\rangle,
$$

where $[a, b]=c,[a, c]=[b, c]=1,|a|=\infty,|b|=\infty,|c| \leq \infty$;

$$
G \cong\left(P_{1} \times P_{2} \times \cdots \times P_{i} \times \cdots \times P_{n}\right) \rtimes\langle b\rangle, \quad n \geq 1,
$$

where, for $i=1, \ldots, n$, the component $P_{i}$ is a $p_{i}$-group, $p_{i} \neq p_{j}$ for $i \neq j,|b|=\infty$, and $P_{i} \rtimes\langle b\rangle$ is of type (2.1), (2.2), (2.3), and (2.4) of Proposition 2.1.

Proof. Let $G$ be an infinite non-abelian 2-generator group of nilpotency class two. Then, $G=A\langle b\rangle$ where $A=\langle c\rangle\langle a\rangle, c=[a, b], A$ is abelian and normal in $G$, and $G=$ $\langle a, b\rangle$. Furthermore, $G^{\prime}=\langle[a, b]\rangle$. Consider $G / Z(G)$. Then either $G / Z(G)$ is torsion free or $G / Z(G)$ has a nontrivial element of finite order. In case $G / Z(G)$ is torsion free, we can show that $\left|G^{\prime}\right|=\infty$. So $G \cong \mathscr{H}$ and is of type (2.5) with $|c|=\infty$.

Now, suppose $G / Z(G)$ has a nontrivial element of finite order. It follows that $G / Z(G)$ is finite which implies $\left|G^{\prime}\right|<\infty$. Then either $G / G^{\prime}$ is torsion free or not. In the first 
case, $G / G^{\prime} \cong \mathbb{Z} \times \mathbb{Z}$ and $G$ is a group of type (2.5) with $|c|<\infty$, and in the second case, $G / G^{\prime} \cong \mathbb{Z}_{m} \times \mathbb{Z}$. Without loss of generality, assume that $|\langle b\rangle|=\infty$ and $|\langle a\rangle|<\infty$, otherwise, relabel the generators so that $G=\langle a, b\rangle$, with $|b|=\infty$ and $A=\langle a, c\rangle,|A|<\infty$. Then $|b|=\infty$ implies that $A \cap\langle b\rangle=\langle 1\rangle$, and we have $G=A \rtimes\langle b\rangle$.

Now, since $A=\langle c, a\rangle$ is a finite abelian group, $A=P_{1} \times \cdots \times P_{i} \times \cdots \times P_{n}$, where $P_{i}$ is a Sylow $p_{i}$-subgroup of $A$. Let $\langle a\rangle=\left\langle a_{1}\right\rangle \times \cdots \times\left\langle a_{i}\right\rangle \times \cdots \times\left\langle a_{n}\right\rangle,\langle c\rangle=\left\langle c_{1}\right\rangle \times \cdots \times\left\langle c_{n}\right\rangle$, with $a_{i}, c_{i} \in P_{i}$ and $c_{i}=\left[a_{i}, b\right]$. We have $P_{i}=\left\langle a_{i}, c_{i}\right\rangle$ and $P_{i}$ is normal in $G$. Thus, there are subgroups $H_{i}=P_{i} \rtimes\langle b\rangle$ of $G$ such that $H_{i}=\left\langle a_{i}, b\right\rangle$. If $H_{i}$ is an abelian group, then it follows that $P_{i}=\left\langle a_{i}\right\rangle,\left|a_{i}\right|=p^{\alpha_{i}}$, and $H_{i}=P_{i} \times\langle b\rangle$. If $H_{i}$ is a non-abelian group, then $H_{i}$ is one of the groups of type (2.1), (2.2), and (2.3) of Proposition 2.1. Thus, $G$ is the group of type (2.6).

3. The tensor squares of groups of class two. In [1, Proposition 3.3], it was shown that the tensor square for a group of class two is abelian. This fact helps us in using the concept of crossed pairing in our computations. We define it here in the case relevant for non-abelian tensor squares.

DeFinITION 3.1. Let $G$ and $L$ be groups. A function $\phi: G \times G \rightarrow L$ is called a crossed pairing if

$$
\begin{aligned}
& \phi\left(g g^{\prime}, h\right)=\phi\left({ }^{g} g^{\prime},{ }^{g} h\right) \phi(g, h), \\
& \phi\left(g, h h^{\prime}\right)=\phi(g, h) \phi\left({ }^{h} g,{ }^{h} h^{\prime}\right),
\end{aligned}
$$

for all $g, g^{\prime}, h, h^{\prime} \in G$.

Crossed pairings allow us to determine homomorphic images of $G \otimes G$ as follows.

Proposition 3.2 [3]. A crossed pairing $\phi: G \times G \rightarrow L$ determines a unique homomorphism of groups $\phi^{\star}: G \otimes G \rightarrow L$ such that $\phi^{\star}(g \otimes h)=\phi(g, h)$ for all $g, h \in G$.

In this section, we also include two results that will be used in the next section. First, suppose we are given groups $H, G, K$, and $L$, where $G$ and $L$ are homomorphic images of $H$ and $K$, respectively. The following proposition enables us to find a crossed pairing from $G \times G$ to $L$ given a crossed pairing from $H \times H$ to $K$, provided certain conditions are met.

Proposition 3.3 [7]. Let $G, H, K$, and $L$ be groups with $\pi: H \rightarrow G$ an epimorphism, $\varphi: K \rightarrow L$ a homomorphism, and $\Gamma: H \times H \rightarrow K$ a crossed pairing. If $\Gamma(\operatorname{ker} \pi, H)$ and $\Gamma(H, \operatorname{ker} \pi)$ are contained in $\operatorname{ker} \varphi$, then there exists a crossed pairing $\Delta: G \times G \rightarrow L$ for which the following diagram commutes:

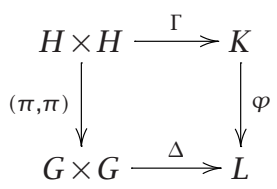

The second result is a lemma on finitely generated abelian groups. The proof is easy and thus omitted here. Observe that we say that a nontrivial element in an infinite cyclic group has order zero. 
LEMMA 3.4. Let $A=\left\langle a_{1}, \ldots, a_{n}\right\rangle$ be a finitely generated abelian group, and let $B=$ $\left\langle b_{1}\right\rangle \times \cdots \times\left\langle b_{n}\right\rangle$ be a direct sum of $n$ cyclic groups, such that the order of $a_{i}$ divides the order of $b_{i}$ for $i=1, \ldots, n$. If $\phi: A \rightarrow B$ is a homomorphism such that $\phi\left(a_{i}\right)=b_{i}$, then $\phi$ is an isomorphism.

4. Computation of the tensor squares. In this section, we determine the tensor squares of the groups classified in Section 2 beginning with the squares of groups of type (2.5).

THEOREM 4.1. Let $G$ be a group of type (2.5). Then

$$
G \otimes G \cong \begin{cases}\mathbb{Z}^{6}, & \text { for }|c|=\infty, \\ \mathbb{Z}^{4} \times \mathbb{Z}_{k}^{2}, & \text { for }|c|=k .\end{cases}
$$

Proof. Let $G$ be a group of type (2.5). If $|c|=\infty$, then $G \cong \mathscr{H}$, and the result follows from [1, Corollary 3.8].

If $|c|=k$, then by [1, Proposition 3.5] it follows that $G \otimes G$ is generated by $a \otimes a$, $b \otimes b, a \otimes b, b \otimes a, a \otimes c$, and $b \otimes c$.

We now establish order bounds for some of the generators of $G \otimes G$. Observing that $c \in Z(G)$, we have $1_{\otimes}=(a \otimes c)^{k}$, and $1_{\otimes}=(b \otimes c)^{k}$. Since $|a|=|b|=\infty$, the first four generators of $G \otimes G$ do not necessarily have finite order, and indeed, it will be shown that they have an infinite order.

Let $g, h \in G$ with $g=a^{m} b^{n} c^{l}$ and $h=a^{m^{\prime}} b^{n^{\prime}} c^{l^{\prime}}$, where $m, m^{\prime}, n, n^{\prime} \in \mathbb{Z}$ and $l, l^{\prime}$ are integers modulo $k$. Let $L=\mathbb{Z}^{4} \times \mathbb{Z}_{k}^{2}$, and denote with $z_{1}, z_{2}, z_{3}, z_{4}$ the components of the four factors of the form $\mathbb{Z}$, and with $z_{5}, z_{6}$ the components of the two factors of the form $\mathbb{Z}_{k}$. Define the mapping $\theta: G \times G \rightarrow L$ componentwise by

$$
\theta(g, h)=\left(z_{1}(g, h), z_{2}(g, h), z_{3}(g, h), z_{4}(g, h), z_{5}(g, h), z_{6}(g, h)\right),
$$

where

$$
\begin{aligned}
& z_{1}(g, h)=m m^{\prime}, \quad z_{2}(g, h)=n n^{\prime}, \quad z_{3}(g, h)=m n^{\prime}, \quad z_{4}(g, h)=n m^{\prime}, \\
& z_{5}(g, h) \equiv n^{\prime}\left(\begin{array}{c}
m \\
2
\end{array}\right)-n\left(\begin{array}{c}
m^{\prime} \\
2
\end{array}\right)+\left(n^{\prime}-n\right) m m^{\prime}+m l^{\prime}-m^{\prime} l \bmod k, \\
& z_{6}(g, h) \equiv m\left(\begin{array}{c}
n^{\prime} \\
2
\end{array}\right)-m^{\prime}\left(\begin{array}{c}
n \\
2
\end{array}\right)+n l^{\prime}-n^{\prime} l \bmod k .
\end{aligned}
$$

Since $m, m^{\prime}, n, n^{\prime}$ are unique integers and $l, l^{\prime}$ are unique modulo $k$, it follows that $\theta$ is well defined. As in [1], $\theta$ is a crossed pairing. By Proposition 3.2, the mapping $\theta$ defined above lifts to a homomorphism $\theta^{\star}$ of $G \otimes G$ onto $L$ such that $\theta^{\star}(g \otimes h)=$ $\theta(g, h)$. In particular, $\theta^{\star}(a \otimes a)=(1,0,0,0,0,0), \theta^{\star}(b \otimes b)=(0,1,0,0,0,0), \theta^{\star}(a \otimes$ $b)=(0,0,1,0,0,0), \theta^{\star}(b \otimes a)=(0,0,0,1,0,0), \theta^{\star}(a \otimes c)=(0,0,0,0,1,0)$, and $\theta^{\star}(b \otimes$ $c)=(0,0,0,0,0,1)$. Thus the generators of $G \otimes G$ map to the generators of $L$. Furthermore, by the order estimates previously established, the order of a generator of $G \otimes G$ divides the order of the corresponding generator of $L$. Thus, by Lemma $3.4, \theta^{\star}$ is an isomorphism and it follows that $G \otimes G \cong \mathbb{Z}^{4} \times \mathbb{Z}_{k}^{2}$ as claimed. 
In order to determine the tensor squares of groups of type (2.6), we first determine the tensor squares of the groups in Proposition 2.1. We start with the groups of type (2.1), dealing with the case $p$ odd and $p=2$ in two separate propositions.

Proposition 4.2. Let $G$ be a group of type (2.1) with $p \neq 2$. Then

$$
G \otimes G \cong \mathbb{Z}_{p^{\alpha}}^{3} \times \mathbb{Z}_{p^{\gamma}}^{2} \times \mathbb{Z}
$$

Proof. Let $G$ be a group of type (2.1) with $p$ prime, $p \neq 2$. It follows that $G \otimes G$ is generated by $a \otimes a, b \otimes b, a \otimes b, b \otimes a, a \otimes c$, and $b \otimes c$. We give now order estimates for 5 of the 6 generators of $G \otimes G$. Using [1, Lemma 3.4], we obtain $1_{\otimes}=(a \otimes c)^{p^{\gamma}}, 1_{\otimes}=$ $b \otimes c^{p^{\gamma}}=(b \otimes c)^{p^{\gamma}}, 1_{\otimes}=a \otimes a^{p^{\alpha}}=(a \otimes a)^{p^{\alpha}}$, and $1_{\otimes}=a^{p^{\alpha}} \otimes b=(a \otimes b)^{p^{\alpha}}(a \otimes c)\left(\begin{array}{c}p_{2}^{\alpha} \\ )\end{array}\right.$. Since $p \neq 2$ and $\alpha \geq \gamma$, we have $p^{\gamma} \mid\left(\begin{array}{c}p^{\alpha} \\ 2\end{array}\right)$. Thus $(a \otimes c)\left(\begin{array}{c}p^{\alpha} \\ 2\end{array}\right)=1_{\otimes}$. We conclude that $1_{\otimes}=(a \otimes b)^{p^{\alpha}}$. Similarly, it follows that $1_{\otimes}=(b \otimes a)^{p^{\alpha}}$. Since $|b|=\infty, b \otimes b$ is not necessarily finite.

Let $g, h \in G$ with $g=a^{m} b^{n} c^{l}$ and $h=a^{m^{\prime}} b^{n^{\prime}} c^{l^{\prime}}$, where $n, n^{\prime} \in \mathbb{Z}, m, m^{\prime}$ are integers modulo $p^{\alpha}$, and $l, l^{\prime}$ are integers modulo $p^{\gamma}$. Let $L=\mathbb{Z}_{p^{\alpha}} \times \mathbb{Z} \times \mathbb{Z}_{p^{\alpha}}^{2} \times \mathbb{Z}_{p^{\gamma}}^{2}$, and denote with $z_{1}, z_{3}, z_{4}$ the components of the three factors of the form $\mathbb{Z}_{p^{\alpha}}$, with $z_{2}$ the component of $\mathbb{Z}$, and with $z_{5}, z_{6}$ the components of the two factors of the form $\mathbb{Z}_{p^{\gamma}}$.

Define the mapping $\theta: G \times G \rightarrow L$ componentwise by

$$
\theta(g, h)=\left(z_{1}(g, h), z_{2}(g, h), z_{3}(g, h), z_{4}(g, h), z_{5}(g, h), z_{6}(g, h)\right),
$$

where

$$
\begin{aligned}
& z_{1}(g, h) \equiv m m^{\prime} \bmod p^{\alpha}, \quad z_{2}(g, h)=n n^{\prime}, \\
& z_{3}(g, h) \equiv m n^{\prime} \bmod p^{\alpha}, \quad z_{4}(g, h) \equiv n m^{\prime} \bmod p^{\alpha}, \\
& z_{5}(g, h) \equiv n^{\prime}\left(\begin{array}{c}
m \\
2
\end{array}\right)-n\left(\begin{array}{c}
m^{\prime} \\
2
\end{array}\right)+\left(n^{\prime}-n\right) m m^{\prime}+m l^{\prime}-m^{\prime} l \bmod p^{\gamma}, \\
& z_{6}(g, h) \equiv m\left(\begin{array}{c}
n^{\prime} \\
2
\end{array}\right)-m^{\prime}\left(\begin{array}{l}
n \\
2
\end{array}\right)+n l^{\prime}-n^{\prime} l \bmod p^{\gamma} .
\end{aligned}
$$

Since $m, m^{\prime}$ are unique modulo $p^{\alpha}, n, n^{\prime}$ are unique integers, and $l, l^{\prime}$ are unique modulo $p^{\gamma}$, and in addition $\alpha \geq \gamma \geq 1$, it follows that $\theta$ is well defined.

Next, we show that the mapping $\theta$ is a crossed pairing. By [1, Proposition 3.7], the mapping $\psi: \mathscr{H} \times \mathscr{H} \rightarrow \mathbb{Z}^{6}$, defined as $\theta$, but $m, n, l, m^{\prime}, n^{\prime}, l^{\prime}$ just being integers, is a crossed pairing. Now, equations (3.1) hold componentwise for $\psi$ as identities in integers. It follows that they hold as congruences modulo any integer. Since, at the same time, the modules given for $z_{1}, z_{3}, z_{4}, z_{5}$, and $z_{6}$ are the largest for which $\theta$ is well defined, we conclude that $\theta$ is a crossed pairing. By Proposition 3.2, the mapping defined above lifts to a unique homomorphism $\theta^{\star}: G \otimes G \rightarrow L$ such that $\theta^{\star}(g \otimes h)=\theta(g, h)$, where the generators of $G \otimes G$, as given above, map to the corresponding generators of $L$. Furthermore, by the order estimates established before, the order of a generator of $G \otimes G$ divides the order of the corresponding generator of $L$. Thus, by Lemma 3.4, it follows that $\theta^{\star}$ is an isomorphism and $G \otimes G \cong \mathbb{Z}_{p^{\alpha}}^{3} \times \mathbb{Z}_{p^{\gamma}}^{2} \times \mathbb{Z}$ as claimed. 
Proposition 4.3. Let $G$ be a group of type (2.1) with $p=2$. Then

$$
G \otimes G \cong \begin{cases}\mathbb{Z}_{2^{\alpha}}^{3} \times \mathbb{Z}_{2^{\gamma}}^{2} \times \mathbb{Z}, & \text { if } \alpha>\gamma, \\ \mathbb{Z}_{2^{\gamma}}^{3} \times \mathbb{Z}_{2^{\gamma+1}} \times \mathbb{Z}_{2 \gamma-1} \times \mathbb{Z}, & \text { if } \alpha=\gamma .\end{cases}
$$

Proof. Let $G$ be a group of type (2.1) with $p=2$. We have two cases, namely, $\alpha>\gamma$ and $\alpha=\gamma$. The first case follows from the proof of Proposition 4.2, setting $p=2$ and observing $\alpha>\gamma$. So we omit the details.

Now, let $\alpha=\gamma$. From [1, Proposition 3.5], it follows that $G \otimes G$ can be generated by $a \otimes a, b \otimes b, a \otimes b,(a \otimes b)(b \otimes a),(a \otimes b)^{2}(a \otimes c)$, and $(a \otimes b)^{2}(b \otimes c)$.

We now establish order bounds for the generators of $G \otimes G$ with the exception of $b \otimes b$. First, notice that the expansion formula as before yields $1_{\otimes}=(a \otimes c)^{2^{\gamma}}$ and $1_{\otimes}=(b \otimes c)^{2^{\gamma}}$. Furthermore, $1_{\otimes}=(a \otimes a)^{2^{\gamma}}, 1_{\otimes}=\left(a^{2^{\gamma}} \otimes b\right)^{2}=(a \otimes b)^{2^{\gamma+1}}(a \otimes c)^{2^{\gamma}}=$ $(a \otimes b)^{2^{\gamma+1}}, 1_{\otimes}=\left(a^{2^{\gamma}} \otimes b\right)\left(b \otimes a^{2^{\gamma}}\right)=((a \otimes b)(b \otimes a))^{2^{\gamma}}, 1_{\otimes}=\left((a \otimes b)^{2}(a \otimes c)\right)^{2^{\gamma-1}}$, and $1_{\otimes}=\left(a^{2^{\gamma}} \otimes b\right)^{2}\left(b \otimes c^{2^{\gamma}}\right)=(a \otimes b)^{2^{\gamma+1}}(b \otimes c)^{2^{\gamma}}=\left((a \otimes b)^{2}(b \otimes c)\right)^{2^{\gamma}}$. Let $\mathscr{H}=\langle x, y\rangle$. Define $\pi: \mathscr{H} \rightarrow G$ by $\pi(h)=a^{m} b^{n} c^{l}$ for $h \in \mathcal{H}$, where $h=x^{m} y^{n} z^{l}, m, n, l \in \mathbb{Z}, z=$ $[x, y]$. It follows that $\pi$ is a homomorphism onto $G$. Next, set $L=\mathbb{Z}_{2^{\alpha}} \times \mathbb{Z} \times \mathbb{Z}_{2^{\gamma+1}} \times \mathbb{Z}_{2^{\gamma}} \times$ $\mathbb{Z}_{2^{\alpha-1}} \times \mathbb{Z}_{2^{\gamma}}$ and $\mathbb{Z}^{6}=\left\langle x_{1}\right\rangle \times \cdots \times\left\langle x_{6}\right\rangle$. Define $\varphi: \mathbb{Z}^{6} \rightarrow L$ by $\varphi=\mu \circ \lambda$, where $\lambda: \mathbb{Z}^{6} \rightarrow \mathbb{Z}^{6}$ is given by $\lambda\left(x_{i}\right)=x_{i}$ for $i=1,2,4,5,6, \lambda\left(x_{3}\right)=x_{3} x_{4}^{-1} x_{5}^{-2} x_{6}^{-2}$, and $\mu: \mathbb{Z}^{6} \rightarrow L$ reduces the generators of $\mathbb{Z}^{6}$ modulo the appropriate powers. Clearly, $\lambda$ is an automorphism and $\mu$ is a homomorphism, so $\varphi$ is a homomorphism with $\varphi\left(x_{i}\right)=\epsilon_{i}, i=1, \ldots, 6$, where $\epsilon_{1}=(1,0,0,0,0,0), \ldots, \epsilon_{6}=(0,0,0,0,0,1) \in L$.

Now, let $\Gamma=\psi$ be the crossed pairing of [1, Proposition 3.7] with $\psi: \mathscr{H} \times \mathscr{H} \rightarrow \mathbb{Z}^{6}$, and $\pi$ the epimorphism of $\mathscr{H}$ onto $G$. By Proposition 3.3, there exists a crossed pairing $\Delta: G \times G \rightarrow L$ for which the diagram commutes, provided

$$
\varphi(\psi(\operatorname{ker} \pi, \mathscr{H}))=\varphi(\psi(\mathscr{H}, \operatorname{ker} \pi))=1
$$

To establish (4.8), let $l=\left(l_{1}, \ldots, l_{6}\right) \in L$ and $h, h^{\prime} \in \mathscr{H}_{\text {with }} h=x^{m} y^{n} z^{l}, h^{\prime}=x^{m^{\prime}} y^{n^{\prime}} z^{l^{\prime}}$, where $m, m^{\prime}, n, n^{\prime}, l, l^{\prime} \in \mathbb{Z}$. By [1, Proposition 3.7] and the definition of $\varphi$, we obtain $\varphi \circ \psi\left(h, h^{\prime}\right)=\varphi\left(x_{1}\left(h, h^{\prime}\right), \ldots, x_{6}\left(h, h^{\prime}\right)\right)=\left(\varphi\left(x_{1}\left(h, h^{\prime}\right)\right), \ldots, \varphi\left(x_{6}\left(h, h^{\prime}\right)\right)\right)=$ $\left(l_{1}\left(h, h^{\prime}\right), \ldots, l_{6}\left(h, h^{\prime}\right)\right)$, where

$$
\begin{aligned}
l_{1}\left(h, h^{\prime}\right) \equiv & m m^{\prime} \bmod 2^{\gamma}, \quad l_{2}\left(h, h^{\prime}\right)=n n^{\prime}, \quad l_{4}\left(h, h^{\prime}\right) \equiv n m^{\prime} \bmod 2^{\gamma}, \\
l_{3}\left(h, h^{\prime}\right) \equiv & m n^{\prime}-m^{\prime} n-2\left(n^{\prime}\left(\begin{array}{c}
m \\
2
\end{array}\right)-n\left(\begin{array}{c}
m^{\prime} \\
2
\end{array}\right)+\left(n^{\prime}-n\right) m m^{\prime}+m l^{\prime}-m^{\prime} l\right) \\
& -2\left(m\left(\begin{array}{c}
n^{\prime} \\
2
\end{array}\right)-m^{\prime}\left(\begin{array}{c}
n \\
2
\end{array}\right)+n l^{\prime}-n^{\prime} l\right) \bmod 2^{\gamma+1}, \\
l_{5}\left(h, h^{\prime}\right) \equiv & n^{\prime}\left(\begin{array}{c}
m \\
2
\end{array}\right)-n\left(\begin{array}{c}
m^{\prime} \\
2
\end{array}\right)+\left(n^{\prime}-n\right) m m^{\prime}+m l^{\prime}-m^{\prime} l \bmod 2^{\gamma-1}, \\
l_{6}\left(h, h^{\prime}\right) \equiv & m\left(\begin{array}{c}
n^{\prime} \\
2
\end{array}\right)-m^{\prime}\left(\begin{array}{c}
n \\
2
\end{array}\right)+n l^{\prime}-n^{\prime} l \bmod 2^{\gamma} .
\end{aligned}
$$


Suppose now that $h \in \operatorname{ker} \pi$, then $m \equiv l \equiv 0 \bmod 2^{\gamma}$ and $n=0$. Thus $l_{1}, l_{2}, l_{4}, l_{5}$, and $l_{6}$ are obviously trivial in $L$. As for $l_{3}$, the terms of the right side can be rearranged as

$$
\begin{aligned}
l_{3}\left(h, h^{\prime}\right) \equiv & 2 m\left(m^{\prime} n-m^{\prime} n^{\prime}-l^{\prime}\right)+2 n\left(\left(\begin{array}{c}
m^{\prime}+1 \\
2
\end{array}\right)-l\right) \\
& +2 l\left(m^{\prime}+n^{\prime}\right)-m^{2} n^{\prime}+n n^{2} m^{\prime} \bmod 2^{\gamma+1} .
\end{aligned}
$$

Since $\alpha=\gamma$, we have $m \equiv l \equiv 0 \bmod 2^{\gamma}$ and $n=0$. Therefore, $2 m \equiv 2 l \equiv 0 \bmod 2^{\gamma+1}$, $2 n=0$, and $m^{2} \equiv 0 \bmod 2^{\gamma+1}$. Thus $l_{3}$ is trivial in $L$. So $\varphi(\psi(\operatorname{ker} \pi, \mathscr{H}))=1$. In a similar manner $\varphi(\psi(\mathscr{H}, \operatorname{ker} \pi))=1$, hence, (4.8) is established.

Since the diagram of Proposition 3.3 commutes for $\Delta$ and $\varphi \circ \psi$ is onto, we conclude that $\Delta$ is onto. Thus, by Proposition 3.2, $\Delta$ lifts to a homomorphism $\Delta^{\star}$ of $G \otimes G$ onto $L$, where the generators of $G \otimes G$ map to the generators of $L$. Furthermore, by the order estimates established before, the order of a generator of $G \otimes G$ divides the order of the corresponding generator of $L$. Thus, by Lemma 3.4, it follows that $\Delta^{\star}$ is an isomorphism and $G \otimes G \cong \mathbb{Z}_{2 \gamma}^{3} \times \mathbb{Z}_{2^{\gamma+1}} \times \mathbb{Z}_{2^{\gamma-1}} \times \mathbb{Z}$ as claimed.

The metacyclic groups of type (2.2) can be viewed as a special case of groups of type (2.3), for which $\sigma=0$, that is, the torsion subgroup has rank one. Thus, the tensor squares of groups of type (2.2) are obtained together with those of type (2.3). Now, we determine the tensor square for a group of type (2.3) and (2.2), dealing with the cases $p \neq 2$ and $p=2$ in two separate propositions.

Proposition 4.4. Let $G$ be a group of type (2.3) or (2.2) with $p \neq 2$. Then

$$
G \otimes G \cong \mathbb{Z}_{p^{\alpha-\gamma+\sigma}}^{2} \times \mathbb{Z}_{p^{\alpha}} \times \mathbb{Z}_{p^{\sigma}}^{2} \times \mathbb{Z}
$$

Proof. Let $G$ be a group of type (2.3) or (2.2) with $p \neq 2$. Set $z=[a, b]$. By [1, Lemma 3.5], it follows that $G \otimes G$ can be generated by $a \otimes a, b \otimes b, a \otimes b,(a \otimes b)(b \otimes a)$, $(a \otimes a)^{p^{\alpha-\gamma}}(a \otimes z)$, and $(a \otimes b)^{p^{\alpha-\gamma}}(b \otimes z)$. Now, we establish order bounds for some of the generators of $G \otimes G$. If we set $z=[a, b]$, then $c=z a^{-p^{\alpha-\gamma}}$ by (2.3). To obtain an order bound for $(a \otimes a)^{p^{\alpha-\gamma}}(a \otimes z)$, expand $c^{p^{\sigma}} \otimes a$ to obtain

$$
1_{\otimes}=c^{p^{\sigma}} \otimes a=(a \otimes a)^{-p^{\alpha-\gamma+\sigma}}(z \otimes a)^{p^{\sigma}}=\left((a \otimes a)^{p^{\alpha-\gamma}}(z \otimes a)^{-1}\right)^{-p^{\sigma}},
$$

thus $\left((a \otimes a)^{p^{\alpha-\gamma}}(a \otimes z)\right)^{p^{\sigma}}=1_{\otimes}$. Similarly,

$$
\left.1_{\otimes}=c^{p^{\sigma}} \otimes b=(a \otimes b)^{-p^{\alpha-\gamma+\sigma}}(a \otimes z)^{\left(p^{\alpha-\gamma+\sigma}\right.}\right)(b \otimes z)^{-p^{\sigma}} .
$$

Since $\alpha+\sigma \geq 2 \gamma$ and $p \neq 2$, this implies that $(a \otimes z)^{\left(\frac{-p^{\alpha-\gamma+\sigma}}{2}\right)}=1_{\otimes}$. Therefore, $\left((a \otimes b)^{p^{\alpha-\gamma}}(b \otimes z)\right)^{p^{\sigma}}=1_{\otimes}$.

Turning now to $(a \otimes b)(b \otimes a)$, expansion of $b \otimes c^{p^{\sigma}}$ leads to

$$
\left.1_{\otimes}=(b \otimes a)^{-p^{\alpha-\gamma+\sigma}}(a \otimes z)^{\left(-p^{\alpha-\gamma+\sigma}\right.}\right)(b \otimes z)^{2^{\sigma}} .
$$

Multiplying (4.13) and (4.12) yields $((a \otimes b)(b \otimes a))^{p^{\alpha-\gamma+\sigma}}=1_{\otimes}$. 
Next, we establish an estimate for the order of $a \otimes b$. By observing $\alpha>\gamma$ and expanding $a^{p^{\alpha}} \otimes b$, we obtain $\left.1_{\otimes}=(a \otimes b)^{p^{\alpha}}(a \otimes z)^{\left(p_{2}^{\alpha}\right.}\right)=(a \otimes b)^{p^{\alpha}}$.

Finally, we turn to the estimate for the order of $a \otimes a$. Here we have

$$
1_{\otimes}=a \otimes c^{p^{\sigma}}=(a \otimes a)^{-p^{\alpha-\gamma+\sigma}}(a \otimes z)^{p^{\sigma}} .
$$

Equating (4.12) and (4.15) yields

$$
(a \otimes z)^{p^{\sigma}}=(a \otimes z)^{-p^{\sigma}}
$$

Obviously, $1_{\otimes}=a^{p^{\alpha}} \otimes z=(a \otimes z)^{p^{\alpha}}$, so $a \otimes z$ has $p$-power order. Hence, we obtain $(a \otimes z)^{p^{\sigma}}=1_{\otimes}$ by (4.16). This, together with (4.12), implies $(a \otimes a)^{p^{\alpha-\gamma+\sigma}}=1_{\otimes}$. Let $\mathcal{H}=$ $\langle x, y\rangle$. Define $\pi: \mathscr{H} \rightarrow G$ by $\pi(h)=a^{m_{1}} b^{m_{2}} c^{l}$, where $h=x^{m_{1}} y^{m_{2}} v^{l}$ with $m_{1}, m_{2}, l \in$ $\mathbb{Z}, v=x^{-p^{\alpha-\gamma}}[x, y]$, and $a, b, c \in G$ as in the proposition. It follows that $\pi$ is an epimorphism. Next, let $L=\mathbb{Z}_{p^{\alpha-\gamma+\sigma}} \times \mathbb{Z} \times \mathbb{Z}_{p^{\alpha}} \times \mathbb{Z}_{p^{\alpha-\gamma+\sigma}} \times \mathbb{Z}_{p^{\sigma}}^{2}$, and $\mathbb{Z}^{6}=\left\langle x_{1}\right\rangle \times \cdots \times\left\langle x_{6}\right\rangle$. Define $\varphi: \mathbb{Z}^{6} \rightarrow L$ by $\varphi=\mu \circ \lambda$, where $\lambda: \mathbb{Z}^{6} \rightarrow \mathbb{Z}^{6}$ is given by $\lambda\left(x_{i}\right)=x_{i}$ for $i=2,4,6$, $\lambda\left(x_{1}\right)=x_{1} x_{5}^{-p^{\alpha-\gamma}} \cdot x_{6}^{-p^{2(\alpha-\gamma)-1}}, \lambda\left(x_{3}\right)=x_{3} x_{4}^{-1} x_{6}^{-p^{\alpha-\gamma}}$, and $\lambda\left(x_{5}\right)=x_{5} x_{6}^{p^{\alpha-\gamma-1}}$, while $\mu: \mathbb{Z}^{6} \rightarrow L$ reduces the generators $\lambda\left(x_{i}\right)$ modulo the appropriate $p$-powers. Specifically, $\varphi\left(x_{i}\right)=\epsilon_{i}, i=1, \ldots, 6$, where $\epsilon_{1}=(1,0,0,0,0,0), \ldots, \epsilon_{6}=(0,0,0,0,0,1) \in L$. It follows that $\lambda$ is an automorphism of $\mathbb{Z}^{6}$ and $\mu$ is a homomorphism of $\mathbb{Z}^{6}$ onto $L$.

Let $h, h^{\prime} \in \mathscr{H}$ with $h=x^{m_{1}} y^{m_{2}} v^{l}$ and $h^{\prime}=x^{m_{1}^{\prime}} y^{m_{2}^{\prime}} v^{l^{\prime}}$, where $v=x^{-p^{\alpha-\gamma}}[x, y]$ as before. Setting $u=[x, y]$, we obtain alternative presentations for $h$ and $h^{\prime}$ as $h=x^{m} y^{n} u^{k}$ and $h^{\prime}=x^{m^{\prime}} y^{n^{\prime}} u^{k^{\prime}}$, where $m=m_{1}-l p^{\alpha-\gamma}, m^{\prime}=m_{1}^{\prime}-l^{\prime} p^{\alpha-\gamma}, n=m_{2}$, $n^{\prime}=m_{2}^{\prime}, k=l$, and $k^{\prime}=l^{\prime}$. By [1, Proposition 3.7], there exists a crossed pairing $\psi: \mathscr{H} \times \mathscr{H} \rightarrow \mathbb{Z}^{6}$, where, in terms of the original presentation,

$$
\begin{aligned}
x_{1}\left(h, h^{\prime}\right)= & \left(m_{1}-l p^{\alpha-\gamma}\right)\left(m_{1}^{\prime}-l^{\prime} p^{\alpha-\gamma}\right), \quad x_{2}\left(h, h^{\prime}\right)=m_{2} m_{2}^{\prime}, \\
x_{3}\left(h, h^{\prime}\right)= & \left(m_{1}-l p^{\alpha-\gamma}\right) m_{2}^{\prime}, \quad x_{4}\left(h, h^{\prime}\right)=\left(m_{1}^{\prime}-l^{\prime} p^{\alpha-\gamma}\right) m_{2}, \\
x_{5}\left(h, h^{\prime}\right)= & m_{2}^{\prime}\left(\begin{array}{c}
m_{1}-l p^{\alpha-\gamma} \\
2
\end{array}\right)-m_{2}\left(\begin{array}{c}
m_{1}^{\prime}-l^{\prime} p^{\alpha-\gamma} \\
2
\end{array}\right)+\left(m_{1}-l p^{\alpha-\gamma}\right) l^{\prime} \\
& -\left(m_{1}^{\prime}-l^{\prime} p^{\alpha-\gamma}\right) l+\left(m_{2}^{\prime}-m_{2}\right)\left(m_{1}-l p^{\alpha-\gamma}\right)\left(m_{1}^{\prime}-l^{\prime} p^{\alpha-\gamma}\right), \\
x_{6}\left(h, h^{\prime}\right)= & \left(m_{1}-l p^{\alpha-\gamma}\right)\left(\begin{array}{c}
m_{2}^{\prime} \\
2
\end{array}\right)-\left(m_{1}^{\prime}-l^{\prime} p^{\alpha-\gamma}\right)\left(\begin{array}{c}
m_{2} \\
2
\end{array}\right)+m_{2} l^{\prime}-m_{2}^{\prime} l .
\end{aligned}
$$

We apply now Proposition 3.3 with $G$ as given in (2.3), $H=\mathscr{H}, K=\mathbb{Z}^{6}$, and $L$ as defined above. For the mappings, let $\varphi=\mu \circ \lambda$ and $\Gamma=\psi$, all as given above, and $\pi: \mathscr{H} \rightarrow$ $G$. By Proposition 3.3, there exists a crossed pairing $\Delta: G \times G \rightarrow L$ such that the diagram (3.2) commutes, provided $\varphi(\psi(\operatorname{ker} \pi, \mathscr{H}))=\varphi(\psi(\mathscr{H}, \operatorname{ker} \pi))=1$. Next, we show that this is the case. Suppose $h, h^{\prime} \in \mathscr{H}^{l}$ where $h=x^{m_{1}} y^{m_{2}} v^{l}$ and $h^{\prime}=x^{m_{1}^{\prime}} y^{m_{2}^{\prime}} v^{l^{\prime}}$. Writing $\varphi \circ \psi: \mathscr{H} \times \mathscr{H} \rightarrow L$ componentwise as $\varphi \circ \psi\left(h, h^{\prime}\right)=\left(l_{1}\left(h, h^{\prime}\right), \ldots, l_{6}\left(h, h^{\prime}\right)\right)$, we 
obtain $l_{i}\left(h, h^{\prime}\right)$ in terms of $x_{i}\left(h, h^{\prime}\right)$ as given above,

$$
\begin{aligned}
& l_{1}\left(h, h^{\prime}\right) \equiv x_{1}\left(h, h^{\prime}\right)-p^{\alpha-\gamma} x_{5}\left(h, h^{\prime}\right)-p^{2(\alpha-\gamma)-1} x_{6}\left(h, h^{\prime}\right) \bmod p^{\alpha-\gamma+\sigma} ; \\
& l_{2}\left(h, h^{\prime}\right)=x_{2}\left(h, h^{\prime}\right) ; \\
& l_{3}\left(h, h^{\prime}\right) \equiv x_{3}\left(h, h^{\prime}\right)-x_{4}\left(h, h^{\prime}\right)-p^{\alpha-\gamma} x_{6}\left(h, h^{\prime}\right) \bmod p^{\alpha} ; \\
& l_{4}\left(h, h^{\prime}\right) \equiv x_{4}\left(h, h^{\prime}\right) \bmod p^{\alpha-\gamma+\sigma} ; \\
& l_{5}\left(h, h^{\prime}\right) \equiv x_{5}\left(h, h^{\prime}\right)+p^{\alpha-\gamma-1} x_{6}\left(h, h^{\prime}\right) \bmod p^{\sigma} ; \\
& l_{6}\left(h, h^{\prime}\right) \equiv x_{6}\left(h, h^{\prime}\right) \bmod p^{\sigma} .
\end{aligned}
$$

If $h \in \operatorname{ker} \pi$, then $m_{1} \equiv 0 \bmod p^{\alpha}, m_{2}=0$ and $l \equiv 0 \bmod p^{\sigma}$. It follows directly from the definition of $x_{i}\left(h, h^{\prime}\right)$ that $l_{i}\left(h, h^{\prime}\right)=0$ for $i=1, \ldots, 6$. Thus $\varphi(\psi(\operatorname{ker} \pi, \mathscr{H}))=1$, as claimed. Similarly, it can be shown that $\varphi(\psi(\mathscr{H}, \operatorname{ker} \pi))=1$. Thus $\Delta$ is a crossed pairing.

Since the diagram of Proposition 3.3 commutes for $\Delta$ and $\psi \circ \varphi$ is onto, we conclude that $\Delta$ is onto. Thus, by Proposition 3.2, $\Delta$ lifts to a homomorphism $\Delta^{\star}$ of $G \otimes G$ onto $L$, where the generators of $G \otimes G$ map to the corresponding generators of $L$. Furthermore, by the order estimates established before, the order of a generator of $G \otimes G$ divides the order of the corresponding generator of $L$. Thus, by Lemma 3.4, it follows that $\Delta^{\star}$ is an isomorphism and $G \otimes G \cong \mathbb{Z}_{p^{\alpha-\gamma+\sigma}}^{2} \times \mathbb{Z}_{p^{\alpha}} \times \mathbb{Z}_{p^{\sigma}}^{2} \times \mathbb{Z}$ as claimed.

Proposition 4.5. Let $G$ be a group of type (2.3) or (2.2) with $p=2$. Then

$$
G \otimes G \cong \mathbb{Z}_{2^{\alpha-\gamma+\sigma+1}} \times \mathbb{Z}_{2^{\alpha-\gamma+\sigma}} \times \mathbb{Z}_{2^{\alpha}} \times \mathbb{Z}_{2^{\sigma}}^{2} \times \mathbb{Z} .
$$

Proof. Let $G$ be a group of type (2.3) or (2.2) with $p=2$. Set $z=[a, b]$. By [1, Proposition 3.5], it follows that $G \otimes G$ can be generated by $a \otimes a, b \otimes b, a \otimes b,(a \otimes$ $b)(b \otimes a),(a \otimes a)^{2^{\alpha-\gamma}}(a \otimes z)$, and $(a \otimes b)^{2^{\alpha-\gamma}}(a \otimes z)^{-2^{\alpha-\gamma-1}}(b \otimes z)$.

Now, we establish order bounds for some of the generators of $G \otimes G$. Notice that $c=z a^{-2^{\alpha-\gamma}}$ by (2.3). Following along the lines of the proof of Proposition 4.4, we get

$$
1_{\otimes}=c^{2^{\sigma}} \otimes a=(a \otimes a)^{-2^{\alpha-\gamma+\sigma}}(a \otimes z)^{-2^{\sigma}},
$$

which gives $\left((a \otimes a)^{2^{\alpha-\gamma}}(a \otimes z)\right)^{2^{\sigma}}=1_{\otimes}$. Similarly,

$$
1_{\otimes}=c^{2^{\sigma}} \otimes b=(a \otimes b)^{-2^{\alpha-\gamma+\sigma}}(a \otimes z)^{2^{\alpha-\gamma+\sigma-1}}(b \otimes z)^{-2^{\sigma}} .
$$

Thus $\left((a \otimes b)^{2^{\alpha-\gamma}}(a \otimes z)^{-2^{\alpha-\gamma-1}}(b \otimes z)\right)^{2^{\sigma}}=1_{\otimes}$. Expansion of $b \otimes c^{2^{\sigma}}$ leads to

$$
1_{\otimes}=(b \otimes a)^{-2^{\alpha-\gamma+\sigma}}(a \otimes z)^{-2^{\alpha-\gamma+\sigma-1}}(b \otimes z)^{2^{\sigma}} .
$$

Multiplying (4.21) and (4.22) yields $((a \otimes b)(b \otimes a))^{2^{\alpha-\gamma+\sigma}}=1_{\otimes}$.

Next, we give an estimate for the order of $a \otimes b$. Since $\alpha>\gamma$, expansion of $a^{2^{\alpha}} \otimes b$ yields $1_{\otimes}=(a \otimes b)^{2^{\alpha}}(a \otimes z)^{2^{\alpha-1}}=(a \otimes b)^{2^{\alpha}}$.

Finally, we estimate the order of $a \otimes a$. We obtain

$$
1_{\otimes}=a \otimes c^{2^{\sigma}}=(a \otimes a)^{-2^{\alpha-\gamma+\sigma}}(a \otimes z)^{2^{\sigma}} .
$$


Equating (4.21) and (4.23) yields $(a \otimes z)^{2^{\sigma+1}}=1_{\otimes}$. Thus, squaring (4.20) leads to $(a \otimes$ a) ${ }^{2^{\alpha-\gamma+\sigma+1}}=1_{\otimes}$.

The rest of the proof follows directly from the proof of Proposition 4.4, with $p$ replaced by 2 and taking $l_{i}\left(h, h^{\prime}\right)$ modulo the appropriate powers of 2 . Thus

$$
G \otimes G \cong \mathbb{Z}_{2^{\alpha-\gamma+\sigma+1}} \times \mathbb{Z}_{2^{\alpha-\gamma+\sigma}} \times \mathbb{Z}_{2 \alpha} \times \mathbb{Z}_{2 \sigma}^{2} \times \mathbb{Z},
$$

as claimed.

For easier reference, we list the tensor squares of groups of type (2.2), the case $\sigma=0$, of Propositions 4.4 and 4.5 in the next corollary.

COROLLARY 4.6. Let $G$ be a group of type (2.2). Then

$$
G \otimes G \cong \begin{cases}\mathbb{Z}_{p^{\alpha-\gamma}}^{2} \times \mathbb{Z}_{p^{\alpha}} \times \mathbb{Z}, & \text { if } p \neq 2, \\ \mathbb{Z}_{2^{\alpha-\gamma+1}} \times \mathbb{Z}_{2^{\alpha-\gamma}} \times \mathbb{Z}_{2^{\alpha}} \times \mathbb{Z}, & \text { if } p=2 .\end{cases}
$$

Our concluding theorem (Theorem 4.7) determines the tensor square of a group of type (2.6). To that end, we mention again that the non-abelian tensor square of an abelian group is just the standard abelian tensor. Thus, for groups of type (2.4), we have $G \otimes G \cong G \otimes_{\mathbb{Z}} G$.

THEOREM 4.7. Let $G$ be a group of type (2.6), that is,

$$
G=\left(P_{1} \times P_{2} \times \cdots \times P_{n}\right) \rtimes\langle b\rangle, \quad n \geq 1,
$$

where, for $i=1, \ldots, n$, the component $P_{i}$ is a $p_{i}$-group, $p_{i} \neq p_{j}$ for $i \neq j,|b|=\infty$, and $G_{i}=P_{i} \rtimes\langle b\rangle$ is of type (2.1), (2.2), (2.3), and (2.4) of Proposition 2.1. Then

$$
G \otimes G \cong T\left(G_{1} \otimes G_{1}\right) \times \cdots \times T\left(G_{n} \otimes G_{n}\right) \times \mathbb{Z},
$$

where $T\left(G_{i} \otimes G_{i}\right)$ is the torsion subgroup of $G_{i} \otimes G_{i}$.

Proof. Let $G$ be a group of type (2.6). Observe that $T(G)=P_{1} \times \cdots \times P_{n}$ is abelian, and if $(|g|,|h|)=1$, then $g \otimes h=1_{\otimes}$.

We prove our claim by induction on $n$, the number of Sylow $p$-subgroups of $T(G)$. If $n=1$, then $G=P_{1} \rtimes\langle b\rangle$ and $G \otimes G \cong T(G \otimes G) \times \mathbb{Z}$ by Propositions 4.2, 4.3, 4.4, and 4.5 .

Suppose $n \geq 2$. Then $G=(P \times Q) \rtimes\langle b\rangle$ with $P=P_{1}$ and $Q=P_{2} \times \cdots \times P_{n}$. Set $U=$ $P \rtimes\langle b\rangle$ and $W=Q \rtimes\langle b\rangle$. Then, for any $g, h \in G$, there exist $u, u^{\prime} \in P \subseteq U, w, w^{\prime} \in W$ such that $g=u w$ and $h=u^{\prime} w^{\prime}$. By expanding $g \otimes h$,

$$
g \otimes h=u w \otimes u^{\prime} w^{\prime}=X(g, h) \cdot\left(w \otimes w^{\prime}\right),
$$

where

$$
X(g, h)=\left(u \otimes u^{\prime}\right)\left(u \otimes w^{\prime}\right)\left(w \otimes u^{\prime}\right)\left([u, w] \otimes u^{\prime}\right)\left([u, w] \otimes w^{\prime}\right)\left(w \otimes\left[u, w^{\prime}\right]\right) .
$$

For $w, w^{\prime} \in W$, there exist $v, v^{\prime} \in Q$ and integers $s, t$ such that $w=v b^{s}$ and $w^{\prime}=v^{\prime} b^{t}$. We substitute $w=v b^{s}$ and $w^{\prime}=v^{\prime} b^{t}$ into (4.29), expand, observing that 
elements in $T(G)$ commute and $g \otimes h=1_{\otimes}$ for $g, h \in T(G)$ with $(|g|,|h|)=1$. We then arrive at

$$
\begin{aligned}
X(g, h)= & \left(u \otimes u^{\prime}\right)(u \otimes b)^{t}(b \otimes[u, b])^{\left(\begin{array}{l}
t \\
2
\end{array}\right)}\left(b \otimes u^{\prime}\right)^{s}(b \otimes[b, u])^{\left(\begin{array}{c}
s \\
2
\end{array}\right)}\left([u, b] \otimes u^{\prime}\right)^{s} \\
& \cdot([u, b] \otimes b)^{s t}(b \otimes[u, b])^{t} .
\end{aligned}
$$

We observe $X(g, h) \in T(U \otimes U)$, hence $\langle X(g, h) \mid g, h \in G\rangle \leq T(U \otimes U)$. On the other hand, $T(U \otimes U) \leq\left\langle u_{1} \otimes u_{2}, u_{3} \otimes b, b \otimes u_{4} \mid u_{1}, u_{2}, u_{3}, u_{4} \in T(U)\right\rangle$. However, for suitable choices of $g$ and $h$, observe that all the above generators are in $\{X(g, h) ; g, h \in G\}$. We conclude that $T(U \otimes U)=\langle X(g, h) \mid g, h \in G\rangle$. The above, together with (4.28), implies $G \otimes G=\langle T(U \otimes U), W \otimes W\rangle$. Observing $(|T(U \otimes U)|,|T(W \otimes W)|)=1$, it follows that $G \otimes G=T(U \otimes U) \times(W \otimes W)$. Since $|Q|=|T(W)|$ has only $n-1$ distinct prime divisors, the claim follows by induction on $n$.

\section{REFERENCES}

[1] M. R. Bacon and L.-C. Kappe, The nonabelian tensor square of a 2-generator p-group of class 2, Arch. Math. (Basel) 61 (1993), no. 6, 508-516.

[2] J. R. Beuerle and L.-C. Kappe, Infinite metacyclic groups and their non-Abelian tensor squares, Proc. Edinburgh Math. Soc. (2) 43 (2000), no. 3, 651-662.

[3] R. Brown, D. L. Johnson, and E. F. Robertson, Some computations of nonabelian tensor products of groups, J. Algebra 111 (1987), no. 1, 177-202.

[4] R. Brown and J.-L. Loday, Excision homotopique en basse dimension [Homotopical excision in low dimension], C. R. Acad. Sci. Paris Sér. I Math. 298 (1984), no. 15, 353-356 (French).

[5] _ Van Kampen theorems for diagrams of spaces, Topology 26 (1987), no. 3, 311-335.

[6] L.-C. Kappe, Nonabelian tensor products of groups: the commutator connection, Groups St. Andrews 1997 in Bath, II, London Mathematical Society Lecture Note Series, vol. 261, Cambridge University Press, Cambridge, 1999, pp. 447-454.

[7] L.-C. Kappe, M. P. Visscher, and N. H. Sarmin, Two-generator two-groups of class two and their nonabelian tensor squares, Glasgow Math. J. 41 (1999), no. 3, 417-430.

Nor Haniza SARmin: Mathematics Department, Faculty of SCIEnCE, Universiti TEKNOLOGI MALAYSIA (UTM), 81310 SKUDAI, JOHOR, MALAYSIA

E-mail address: nhs@me12.fs . utm.my 


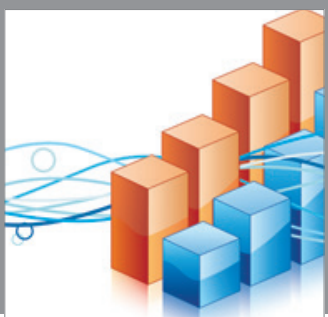

Advances in

Operations Research

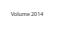

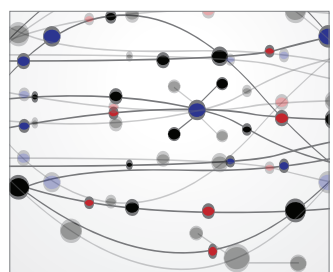

\section{The Scientific} World Journal
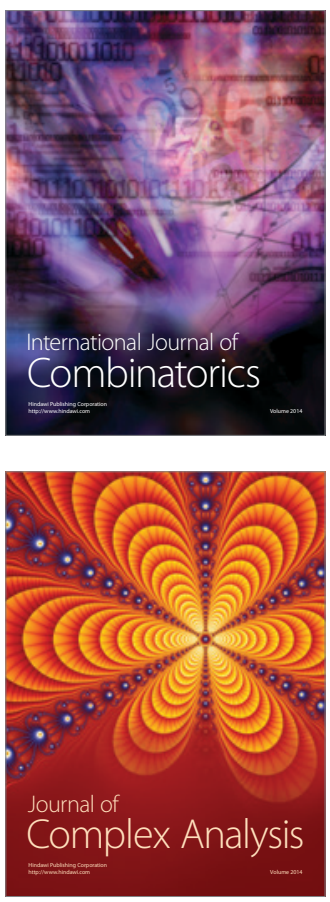

International Journal of

Mathematics and

Mathematical

Sciences
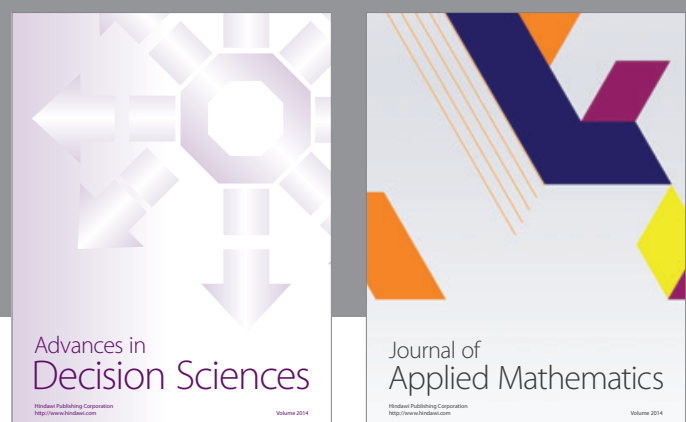

Journal of

Applied Mathematics
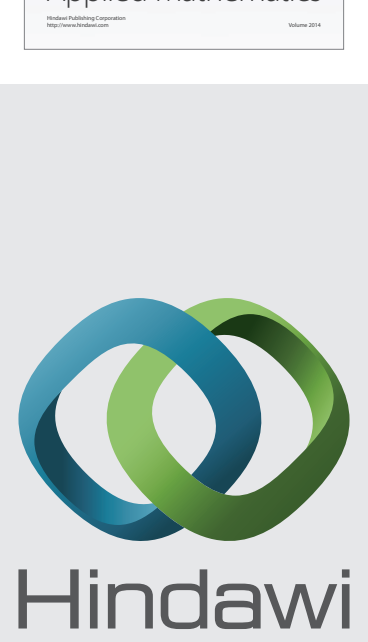

Submit your manuscripts at http://www.hindawi.com
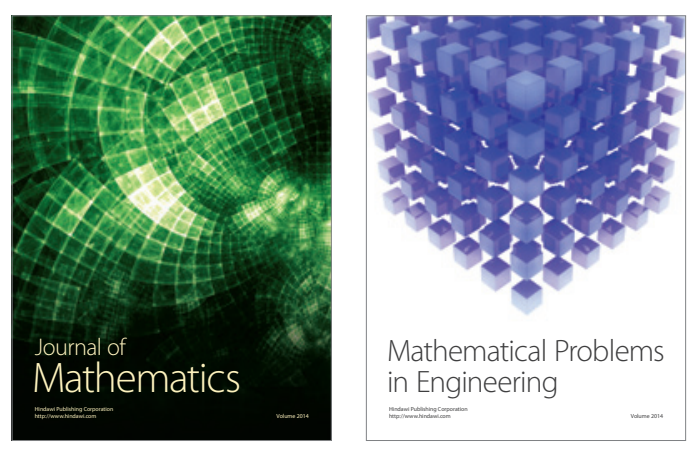

Mathematical Problems in Engineering
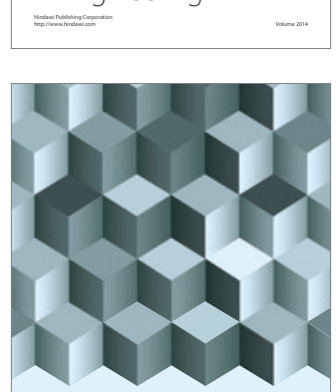

Journal of

Function Spaces
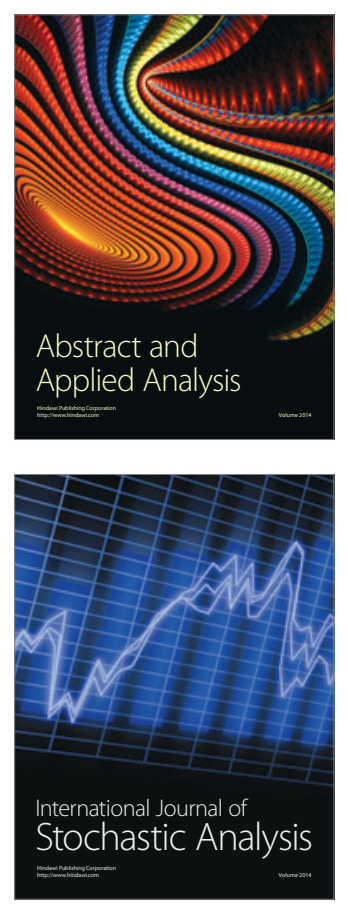

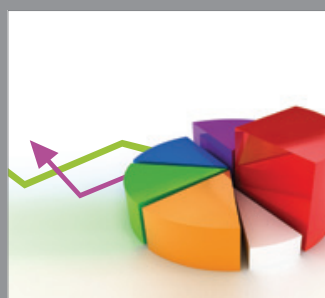

ournal of

Probability and Statistics

Promensencen
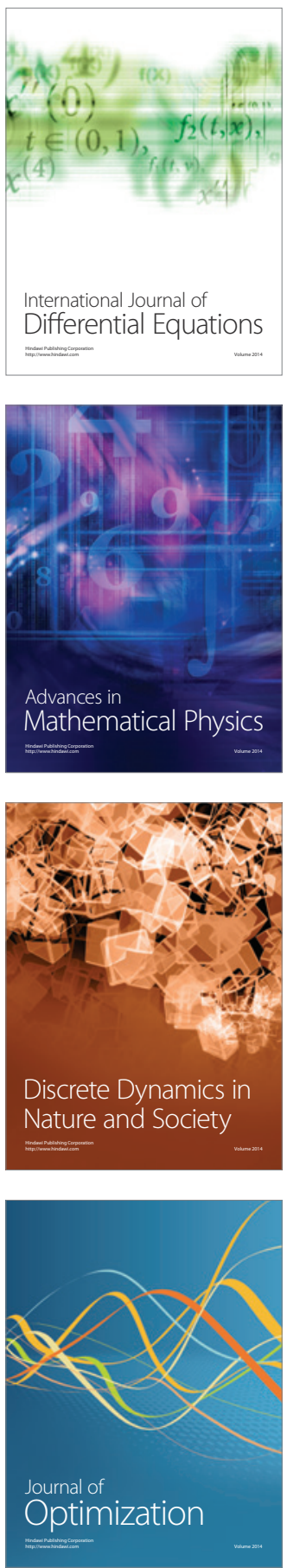